\title{
Aharonov-Bohm oscillations in a mesoscopic ring with asymmetric arm-dependent injection
}

\author{
P. Vasilopoulos, ${ }^{1,2}$ O. Kálmán, ${ }^{2,3}$ F. M. Peeters, ${ }^{2}$ and M. G. Benedict ${ }^{3}$ \\ ${ }^{1}$ Department of Physics, Concordia University, 1455 de Maisonneuve Ouest, Montréal, Quebec, Canada H3G 1 M8 \\ ${ }^{2}$ Departement Fysica, Universiteit Antwerpen (Campus Groenenborger) Groenenborgerlaan 171 B-2020 Antwerpen, Belgium \\ ${ }^{3}$ Department of Theoretical Physics, University of Szeged, Tisza Lajos körút 84 H-6720 Szeged, Hungary \\ (Received 31 January 2006; revised manuscript received 3 November 2006; published 4 January 2007)
}

\begin{abstract}
Electron transport through mesoscopic, one-dimensional rings with asymmetric injection into the arms of the ring is studied, in the presence of a Aharonov-Bohm flux, by means of an appropriate $\mathbf{S}$ matrix. This matrix is expressed in terms of two parameters, one of which $(\lambda)$ accounts phenomenologically for this asymmetric injection into the arms of the ring. In addition, the effect of a scatterer placed in one arm of the ring is considered. Explicit expressions are obtained for the transmission as a function of the incident electron energy, the magnetic field, the asymmetry parameter $\lambda$, and the strength of the scatterer. Results of the literature for symmetric rings are described by $\lambda=1$ and readily recovered. We relate our results to rings of finite width.
\end{abstract}

DOI: 10.1103/PhysRevB.75.035304

\section{INTRODUCTION}

The study of rings goes back to Aharonov and Bohm (AB) who demonstrated the importance of vector potentials in quantum mechanics. ${ }^{1}$ This goes under the name $\mathrm{AB}$ effect and one frequently uses the term $\mathrm{AB}$ oscillations for the oscillations in the resistance of a ring as a function of the flux penetrating the interior of the ring. Another major study is now referred to as the Aharonov-Casher ${ }^{2}$ effect, which is similar to the $\mathrm{AB}$ effect but it is due to the spin-orbit interaction (SOI). Other developments concern the Berry phase. ${ }^{3}$ With the development of new fabrication techniques and the size reduction of samples, rings are now very intensely studied, especially in connection with the SOI (see Refs. 4 and 5 and references cited therein).

In a quantum ring of finite width the connection between the current-carrying leads and the ring can be complicated and may lead to reflection at the lead-ring junction and to asymmetric injection in the two arms of the ring. This asymmetry can be a consequence of the difference in length between the upper and lower arms or of fabrication defects but it can also be induced by a magnetic field as a consequence of the Lorentz force. Such an asymmetry was demonstrated recently from a pure numerical treatment of the transmission through a finite-width lead-ring system; it led to incomplete $\mathrm{AB}$ oscillations due to partially destructive interferences. ${ }^{6}$

To our knowledge all previous works that study transmission through a ring employ an $\mathbf{S}$ matrix that is symmetric with respect to both arms of the ring ${ }^{7-10}$. Though this may not be as restrictive as it sounds, it is more realistic to reexamine the problem using an $\mathbf{S}$ matrix that is not symmetric with respect to both arms of the ring and possibly make a connection with the asymmetry mentioned above. ${ }^{6}$

An asymmetry can be introduced by placing, e.g., one scatterer in one arm of the ring or by locally applying a gate that affects the properties of one arm. The scatterer may introduce important phase shifts in the electron wave function and change drastically the position and/or amplitude of the $\mathrm{AB}$ oscillations. This has been thoroughly investigated theoretically ${ }^{7}$ and experimentally. ${ }^{8}$ Although we will consider such a case, here we are mainly concerned with asym- metric current injection into the arms of the ring through one of the leads, cf. Fig. 1.

In view of the above, we propose a one-dimensional (1D) model in which asymmetries due to fabrication, scatterers, and especially asymmetric current injection through one of the leads are parametrized by a small number of parameters but which leads to explicit analytical results. This has the advantage of being more useful to experimentalists than the pure numerical treatment of Ref. 6.

In the next section we formulate the problem and derive analytic expressions for the transmission amplitude. We present analytical results in Sec. III and numerical results in Secs. IV and V. Concluding remarks follow in Sec. VI.

\section{FORMULATION OF THE PROBLEM}

At each junction of a lead with the ring, indicated by the triangles in Fig. 1, we have three outgoing waves with amplitudes $\left(\alpha^{\prime}, \beta^{\prime}, \gamma^{\prime}\right)=\boldsymbol{\alpha}^{\prime}$ and three incoming waves with am-

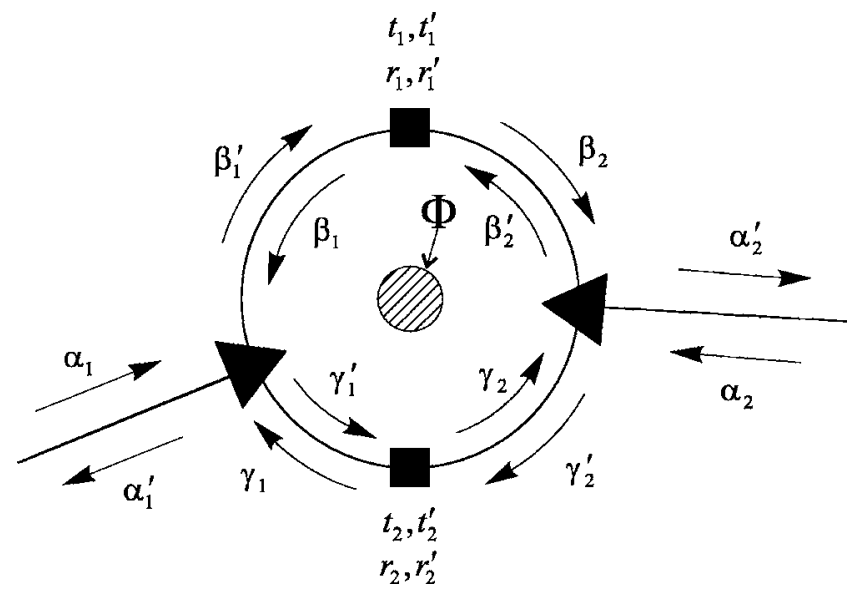

FIG. 1. A ring of radius $R$ connected to two leads, indicated by triangular arrows, with two elastic scatterers indicated by the black squares. The transmission and reflection amplitudes of the scatterers are denoted by $t_{i}$ and $r_{i}, i=1,2$ respectively. A flux $\Phi$ pierces the ring through its center. 
plitudes $(\alpha, \beta, \gamma)=\boldsymbol{\alpha}$. They are related by a $3 \times 3 \mathbf{S}$ matrix in the manner

$$
\boldsymbol{\alpha}^{\prime}=\mathbf{S} \boldsymbol{\alpha} .
$$

Current conservation implies that $\mathbf{S}$ is unitary and timereversal invariance, when applicable, entails $\mathbf{S}^{*}=\mathbf{S}^{-1}$. This means that the $\mathbf{S}$ matrix is symmetric. ${ }^{9}$ Further, we assume that it is real. Then $\mathbf{S}$ is given by

$$
S=\left[\begin{array}{lll}
a & b & c \\
b & d & e \\
c & e & f
\end{array}\right] .
$$

If the $\mathbf{S}$ matrix is symmetric with respect to both arms, one takes $b=c$ and $d=f$ [see Fig. 1 and, e.g., Eqs. (A1) and (A2)]. We introduce an asymmetry by taking $b=\lambda c$. Then, as detailed in Appendix A, the unitarity of the $\mathbf{S}$ matrix leads to the following form:

$$
S=\left[\begin{array}{ccc}
a & \lambda \nu & \nu \\
\lambda \nu & \eta-a & -\lambda \eta \\
\nu & -\lambda \eta & 1-\eta
\end{array}\right],
$$

where $\nu=\left(1-a^{2}\right)^{1 / 2} / \mu, \eta=(a+1) / \mu^{2}, \mu=\left(\lambda^{2}+1\right)^{1 / 2}$, and -1 $<a<1$. Equation (3) is also valid for $\lambda=1$ and the determinant of $\mathbf{S}$ is invariant under the change $\lambda \rightarrow 1 / \lambda$. This change reflects the fact that the results should be the same when the asymmetric injection favors equally the upper or lower arm.

The reflection probability at the left junction is $a^{2}$. Perfect reflection entails $a=1$ and perfect transmission $a=0$. These two limits correspond to those of $\epsilon$ in Ref. 9 being, respectively, 0 and $1 / 2$. It can be invoked that the probability of perfect reflection at the left junction should be independent of the asymmetry of the ring with respect to the two arms. Therefore, one should have $a^{2}=1-2 \epsilon$. It will be shown later in a different way that indeed one has $a^{2}=1-2 \epsilon$.

\section{TRANSMISSION AMPLITUDE}

Equation (1) relates the amplitudes of the incoming waves to those of the outgoing ones. For the usual scattering from the left we take $\alpha_{1}=1$ and $\alpha_{2}=0$. The corresponding transmission is given by $T=\left|\alpha_{2}^{\prime}\right|^{2}$. We proceed along the lines of Ref. 9. We write Eq. (1) as $\boldsymbol{\alpha}_{2}^{\prime}=\mathbf{S} \boldsymbol{\alpha}_{2}$ for the right junction and as $\boldsymbol{\alpha}_{1}^{\prime}=\mathbf{S} \boldsymbol{\alpha}_{1}$ for the left junction. The connection between the two junctions is made by writing

$$
\left(\begin{array}{l}
\beta_{2} \\
\beta_{2}^{\prime}
\end{array}\right)=e^{-i \theta_{1}} \mathbf{t}_{1}\left(\begin{array}{l}
\beta_{1}^{\prime} \\
\beta_{1}
\end{array}\right)
$$

for the amplitudes in the upper arm, where $\mathbf{t}_{1}$ is the matrix describing the transfer through scatterer 1, (cf. Fig. 1), and $\theta_{1}$ a phase shift introduced by the flux $\Phi=\pi R^{2} B, B$ being the magnetic field and $R$ the ring's radius. The matrix $\mathbf{t}_{1}$ is given by

$$
\mathbf{t}_{1}=\left(\begin{array}{cc}
1 / t^{*} & -r^{*} / t^{*} \\
-r / t & 1 / t
\end{array}\right)
$$

with $r$ and $t$ the reflection and transmission amplitudes, respectively. A similar expression transfers the amplitudes in the lower arm and involves a phase shift $\theta_{2}$. These shifts satisfy the relation $\theta_{1}+\theta_{2}=2 \pi \Phi / \Phi_{0}$, where $\Phi_{0}=h / e$ is the flux quantum. Using these expressions and solving the systems of equations $\boldsymbol{\alpha}_{2}^{\prime}=\mathbf{S} \boldsymbol{\alpha}_{2}$ and $\boldsymbol{\alpha}_{1}^{\prime}=\mathbf{S} \boldsymbol{\alpha}_{1}$, as detailed in Appendix A, we obtain the expression for $\alpha_{2}^{\prime}$.

We will consider only two cases: (i) no scatterers are present in the ring's arms, and (ii) one scatterer is present in one arm. The case with both scatterers present can be treated in the same way (see Ref. 9 for $\lambda=1$ ).

For case (i) we have $t_{1}=t_{2}=e^{i \phi}, r_{1}=r_{2}=r_{1}^{\prime}=r_{2}^{\prime}=0, \theta_{1}=\theta_{2}$ $=\pi \Phi / \Phi_{0}$, and $\phi$ the phase change of the transmitted wave. It is related to the energy $E$ by $^{11} \phi=\left(2 m^{*} E\right)^{1 / 2}(\pi R / \hbar)$. Then, using Eq. (A16) of Appendix A, we obtain

$$
\alpha_{2}^{\prime}=\frac{-2 i\left(a^{2}-1\right) \Lambda e^{-i \theta}\left(\lambda^{2}+e^{2 i \theta}\right) \sin \phi}{(a+1)^{2} \Lambda_{\theta}-\Lambda^{2}[F(\phi, a)+2 a]},
$$

where $\Lambda=\lambda^{2}+1, \Lambda_{\theta}=\lambda^{4}+2 \lambda^{2} \cos 2 \theta+1$, and $F(\phi, a)=\left(a^{2}\right.$ $+1) \cos 2 \phi+i\left(a^{2}-1\right) \sin 2 \phi$. Then the transmission is given by

$$
T=\frac{4\left(a^{2}-1\right)^{2} \Lambda^{2} \Lambda_{\theta} \sin ^{2} \phi}{\left\{(a+1)^{2} \Lambda_{\theta}-\Lambda^{2} a_{\phi}\right\}^{2}+\left(a^{2}-1\right)^{2} \Lambda^{4} \sin ^{2} 2 \phi},
$$

where $a_{\phi}=\left(a^{2}+1\right) \cos 2 \phi+2 a$.

Surprisingly, for $\theta=0$ we have $\Lambda_{\theta}=\Lambda^{2}$ and the dependence on $\lambda$ disappears. Then $\alpha_{2}^{\prime}$ takes the much simpler form $\alpha_{2}^{\prime}=2 i\left(a^{2}-1\right) \sin \phi /\left[F(\phi, a)-\left(a^{2}+1\right)\right]$. This simplifies the transmission considerably, as it takes the form

$$
T=\frac{4\left(a^{2}-1\right)^{2} \sin ^{2} \phi}{\left\{(a+1)^{2}-a_{\phi}\right\}^{2}+\left(a^{2}-1\right)^{2} \sin ^{2} 2 \phi} .
$$

For case (ii) we have $t_{1}=T_{s}^{1 / 2} e^{i \phi}, t_{2}=e^{i \phi}, r_{1}=r_{1}^{\prime}$ $=R_{s}^{1 / 2} e^{-i(\pi / 2-\phi)}, r_{2}=r_{2}^{\prime}=0$, and $\theta_{1}=\theta_{2}=\pi \Phi / \Phi_{0} . T_{s}$ is the transmission amplitude of the scatterer and $R_{s}=1-T_{s}$ is the reflection amplitude. Then $\alpha_{2}^{\prime}$ takes the form

$$
\alpha_{2}^{\prime}=\frac{-2 i\left(a^{2}-1\right) \Lambda e^{i \theta}\left[\left(\lambda^{2} T_{s}^{1 / 2} e^{-2 i \theta}+1\right) \sin \phi-R_{s}^{1 / 2}\right]}{(a+1)^{2} \Lambda_{s \theta}-\Lambda^{2}\left[F(\phi, a)+2 R_{s}^{1 / 2} G(\phi, a) / \Lambda+2 a\right]},
$$

where $\Lambda_{s \theta}=\lambda^{4}+2 \lambda^{2} T_{s}^{1 / 2} \cos 2 \theta+1$ and $G(\phi, a)=\left[\left(a^{2}-2 \lambda a\right.\right.$ $\left.+1) \sin \phi-i\left(a^{2}-1\right) \cos \phi\right]$. For $R_{s} \rightarrow 0$ we have $T_{s}=1$ and Eq. (9) reduces to Eq. (6). In contrast though with Eq. (6), the $\theta=0$ limit of Eq. (9) does depend on $\lambda$. The corresponding expressions though for $\alpha_{2}^{\prime}$ and $T$ are too lengthy and will not be given here.

It is interesting to combine the asymmetric injection represented by $\lambda$ with an asymmetry in the ring's arms due to different average densities. ${ }^{12}$ Without any scatterer in the arms this asymmetry can be modeled by taking $t_{1}=e^{i(\phi+\delta)}$ and $t_{2}=e^{i(\phi-\delta)}$ and the same other parameters as in case (i). The resulting expression for $\alpha_{2}^{\prime}$ can be written as 

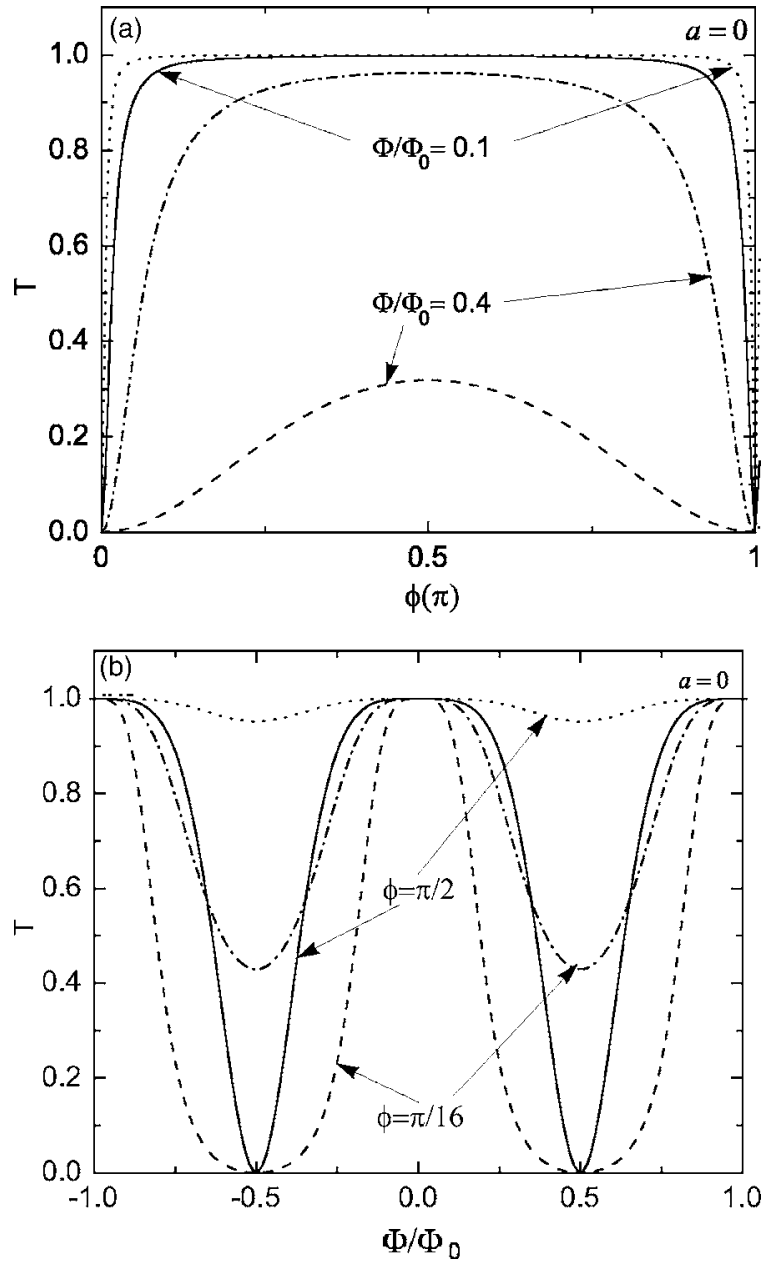

FIG. 2. Transmission vs $\phi$ (a) for two values of the flux and vs flux (b) for two values of $\phi$. The solid and dashed curves are for $\lambda=1$ and the dotted and dash-dotted curves for $\lambda=3$. The value of $a$ is zero, i.e., there is no reflection at the left lead-ring junction.

$$
\alpha_{2}^{\prime}=\frac{-2 i\left(a^{2}-1\right) \Lambda e^{-i \theta}\left[\lambda^{2} \sin (\phi-\delta)+e^{2 i \theta} \sin (\phi+\delta)\right]}{(a+1)^{2} \Lambda_{\theta \delta}-\Lambda^{2}[F(\phi, a)+2 a \cos 2 \delta]+G(a, \delta)}
$$

where $\Lambda_{\theta \delta}=\left(\lambda^{4}+1\right) \cos 2 \delta+2 \lambda^{2} \cos 2 \theta$ and $G(a, \delta)=i\left(a^{2}-1\right)$ $\times\left(\lambda^{4}-1\right) \sin 2 \delta$. For $\delta=0$ Eq. (10) reduces to Eq. (6) while for $\lambda=1$ it gives Eq. (3) of Ref. 12 with which an experimentally observed period halving of the $\mathrm{AB}$ oscillations was explained. Notice further that, in contrast with Eq. (6), the dependence on $\lambda$ does not disappear from Eq. (10) if we set $\theta=0$.

\section{RESULTS}

We now present numerical results for the transmission $T$ given by $T=\left|\alpha_{2}^{\prime}\right|^{2}$. We evaluate $T$ using Eq. (6) and plot it vs $\phi$ in Fig. 2(a) for two different values of the flux. In Fig. 2(b) we plot $T$ as a function of the flux $\Phi / \Phi_{0}$ for two different values of $\phi$. In both panels we have $a=0$. This value of $a$ corresponds to $\epsilon=1 / 2$ in Ref. 9 and the figure is intending to show the effect of the asymmetric injection $(\lambda \neq 1)$ with re-
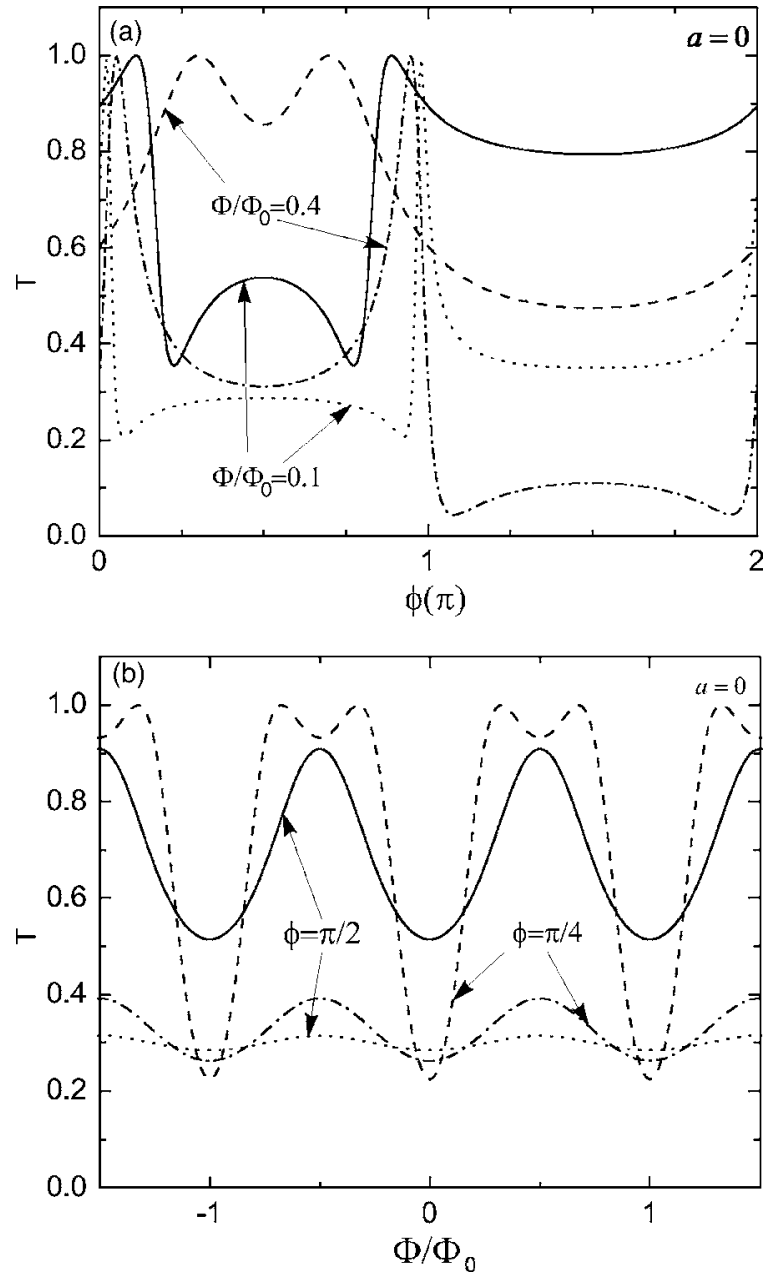

FIG. 3. Transmission vs $\phi$ (a) and vs flux (b) when one scatterer is present in one of the arms with strength $T_{s}=0.25$. The curves are marked as in Fig. 2.

spect to both ring arms: $\lambda=1$ pertains to solid and dashed curves and $\lambda=3$ to dotted and dash-dotted curves. For $\lambda=1$ the results coincide with those of Ref. 9.

If a scatterer is present in one of the arms, we evaluate $T$ using Eq. (9) and plot it in Fig. 3 for $T_{s}=0.25$. Panels and curves are marked as in Fig. 2. As can be seen, a major difference between the two cases is the phase shift introduced by the scatterer in the $T$ vs $\Phi / \Phi_{0}$ panel and the asymmetry between the left and right parts in the $T$ vs $\phi$ panel. The sinusoidal dependence of the transmission on $\phi$ or the flux $\Phi / \Phi_{0}$ stems directly from that of the transmission [cf. Eq. (7)], and especially from that of the numerator for $\lambda$ $>1$.

We now consider only the asymmetric case, fix the value of $\lambda(\lambda=3)$, and focus attention on the dependence of the transmission on the parameter $a$. We show the results in Fig. 4 only for case (i), i.e., when no scatterer is present. When one is present, a phase shift of $\pi / 2$ occurs in the $T$ vs $\phi$ curves and maxima are converted into minima and vice versa. The flux is set to $\Phi / \Phi_{0}=0.4$ in panel (a) and $\phi$ is set to $\pi / 2$ in panel (b). As expected from Eqs. (7) and (9), the transmission $T$ decreases with increasing $a$ when $\phi$ is fixed. 

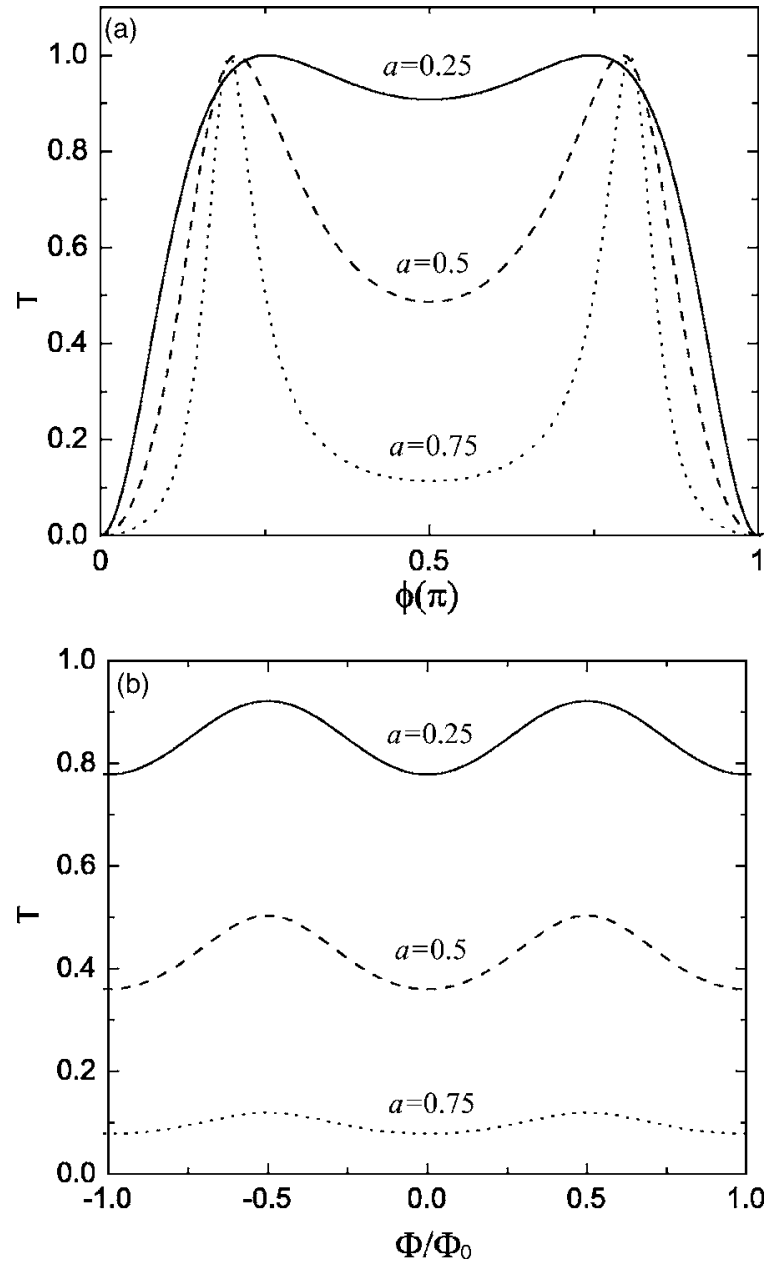

FIG. 4. Transmission vs $\phi$ (a) for $\Phi / \Phi_{0}=0.4$ and vs flux (b) for $\lambda=3$ and $\phi=\pi / 2$ with $a$ as indicated.

The results of Figs. 1-4 show that the parameter $\lambda$, which has to be real for the $S$ matrix to be unitary, affects the amplitude of the transmission but not its phase. We compared the conductance $G$ resulting from Eq. (10) with the experimental results of the right panel of Fig. 3 of Ref. 12. We used $\lambda=1, \alpha=0$ corresponding to $\epsilon=1 / 2$, and the relation of $\delta$ with $k_{F}$ given in this work. Though the agreement is a bit better than that reported in Ref. 12, especially with regard to the oscillation amplitude of $G$ vs $\phi$, determined by the Fermi level or $G$ increase, it remains qualitatively the same and changing $\lambda=1$ to $\lambda \neq 1$, with $\alpha=0$ or $\alpha \neq 0$, brings only a minor quantitative improvement.

\section{COMPARISON WITH OTHER APPROACHES, RINGS OF FINITE WIDTH}

The results presented so far are valid for rings of zero width or rings whose width is much smaller than their radii and only the lowest (in the radial direction) energy level is occupied. The question then arises: (i) how the results compare to those of other approaches, and (ii) how relevant they are to rings of finite width.

With regard to point (i) one frequently followed approach is to consider the same 1D geometry but employ Griffith's boundary conditions at the junctions between the leads and the ring. ${ }^{5}$ Neither the $\mathbf{S}$-matrix approach nor these conditions apply to rings of finite width. One then has to resort to either pure and often heavily involved numerical calculations that pertain to point (ii) (Refs. 4 and 6) or an appropriate modification of Griffith's conditions. ${ }^{13}$ We first address point (i) and then point (ii).

Comparing the zero magnetic-field limit of Eq. (24) of Ref. 9 for the transmission, $T=\left|\alpha_{2}^{\prime}\right|^{2}=1 /\left[(1-2 \epsilon) \sin ^{2} \phi / \epsilon^{2}\right.$ $+1]$, with the zero SOI limit of $T$ in Ref. 5, $T$ $=1 /\left[9 \sin ^{2} \phi / 16+1\right]$, obtained by applying Griffith's boundary conditions, one finds that the results coincide when the parameter $\epsilon$ is equal to $4 / 9$, which is close to the upper limit $\epsilon=1 / 2$. If we compare any of these expressions with ours, as obtained using Eq. (8), for any $\lambda$ we obtain $a= \pm(1-2 \epsilon)^{1 / 2}$ in the first case and $a= \pm 1 / 3$ in the second.

Surprisingly, the same value of $\epsilon$ is obtained for a nonzero magnetic field if one disregards the fact that $\theta$ in Eq. (24) of Ref. 9 appears as $\theta / 2$ in Ref. 5 since the former is given by $T=4 \sin ^{2} \phi \cos ^{2} \theta /\left[\left(2 a^{2} / \epsilon\right) \sin ^{2} \phi+\left(b^{2} / \epsilon\right)(\cos 2 \theta-\cos 2 \phi]^{2}\right.$, $a^{2}+b^{2}=1-\epsilon$, and the latter by $T$ $=4 \sin ^{2} \phi \cos ^{2}(\theta / 2) /\left[\sin ^{2} \phi / 2+(\cos \theta-\cos 2 \phi]^{2}\right.$. Unfortunately, attempting to make the same comparison between any of these expressions for $T$ and ours, using Eq. (7) with $\lambda$ $\neq \pm 1$, gives a very unwieldy result that involves transcendental equations.

One way to proceed with case (ii), i.e., with rings of finite width, is to slightly modify Griffith's conditions so that the width appears in them and in the expression for the transmission. This is done ${ }^{13}$ at the expense of an additional parameter $\nu$, of order 1 , whose value is obtained from a comparison of the transmission with an exact calculation. We have done so for the transmission through a ring of width $W$ at zero magnetic field and give the result in Appendix B [cf. Eq. (B2)]. Equating this result to that of Ref. 9 gives $\epsilon=4 /(\mu+9)$ with $\mu=4 \nu^{2} / k^{2} W^{2}$, while equating it to ours leads to a value of $a$ determined from $\left(a^{2}+1\right) /\left(a^{2}-1\right)=-[(\mu+1)(\mu+9)+1]^{1 / 2}$. Notice that this determination of $\epsilon$ and $a$ makes them depend on the energy, through the wave vector $k$, and the width $W$. We assume that approximately the same values are obtained for $B \neq 0$.

A second way to proceed is to compare directly our result for $W=0$ with an exact numerical one and try to fit the latter by varying the parameter $\lambda$. We have done so with $\lambda=c /[1$ $\left.+\left(\Phi / \Phi_{0}\right)^{s}\right]$ in order to mimick the asymmetry reported in Ref. 6 and attributed to the effect of the Lorentz force in a quantum wire of finite width. In the top panel of Fig. 5 we show the transmission as a function of the flux. The values of $\phi$ used in producing the solid, dashed, and dotted curve correspond to the wave vectors $k=0.091 / \mathrm{nm}, k=0.06 / \mathrm{nm}$, and $k=0.053 / \mathrm{nm}$ in Fig. 8 of Ref. 6. The values of $a, s$, and $c$ used are $a=0.25, s=1.25$, and $c=1$. The qualitative agreement between the two results is very good with regard to the period of the oscillations and the height of the transmission peaks which in our case are more rounded than in Ref. 6. Notice, in particular, the reduction of the transmission minima with increasing $\Phi / \Phi_{0}$, which is attributed to the influence of the Lorentz force and is well reproduced. Of course our simple model cannot reproduce all the details of 

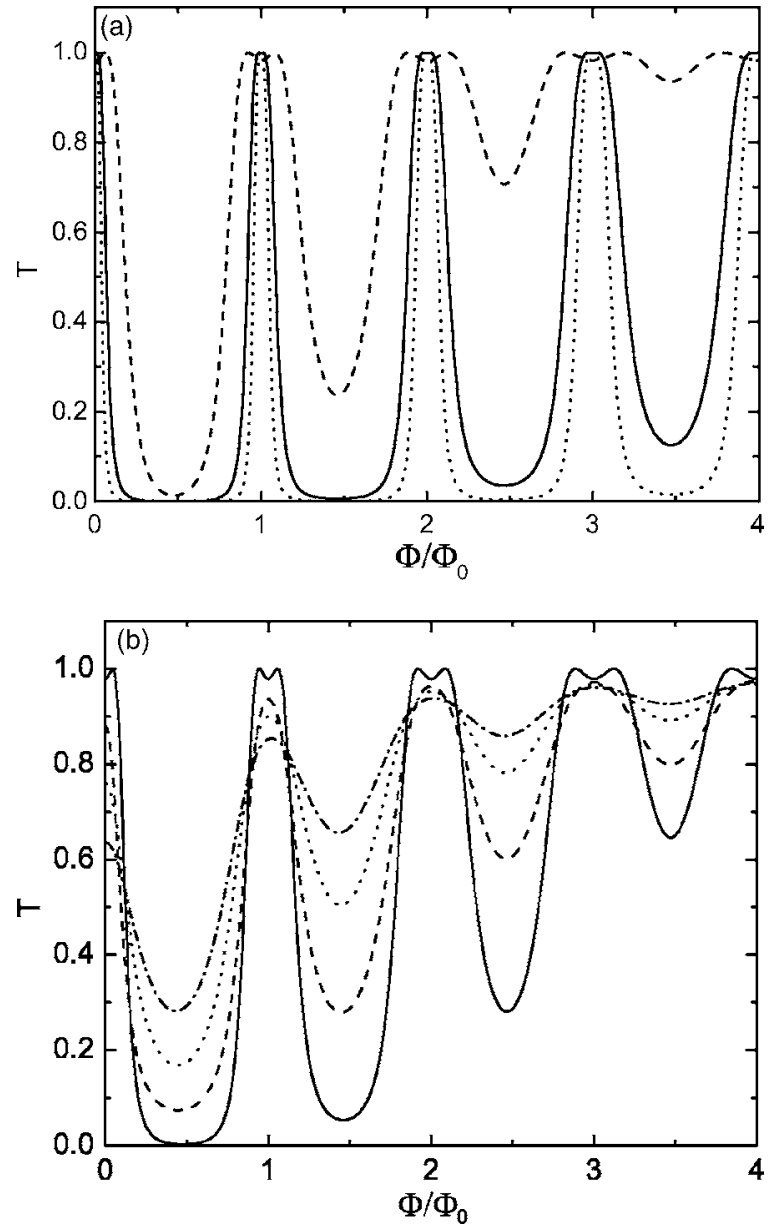

FIG. 5. Top panel: Transmission vs flux with $\phi$ such that the solid, dashed, and dotted curve correspond to the wave vectors $k$ $=0.091 / \mathrm{nm}, k=0.006 / \mathrm{nm}$, and $k=0.053 / \mathrm{nm}$, in Fig. 8 of Ref. 6 . The values of $a, s$, and $c$ used are $a=0.25, s=1.25$, and $c=1$. No scatterer is present in the ring's arms. Bottom panel: As in top panel with one scatterer present in one of the arms. The dashed-dotted, dotted, dashed, and solid curves are for $T_{s}=0.25,0.5,0.75$, and 1 , respectively. The other parameters are $\phi=6.95 \pi, a=0.4, s=1.5$, and $c=1$.

Ref. 6 but its analytical simplicity is an advantage over the approach of Ref. 6.

The asymmetry reported in Ref. 6 is also exhibited in the bottom panel of Fig. 5 where we plot $T$ vs $\Phi / \Phi_{0}$ for case (ii), i.e., when one scatterer is present in one arm. We plot $T$ for different $T_{s}$ using $\phi=6.95 \pi$. The dashed-dotted, dotted, dashed, and solid curves correspond to $T_{s}=0.25,0.5,0.75$, and 1 , respectively. We also used $a=0.4, s=1.25$, and $c=1$. The results are similar to those shown in the top panel, where different wave vectors were used from one curve to another. As can be seen, the overall trend is similar to that of Fig. 12 of Ref. 6. A detailed comparison cannot be made though because here we consider transmission through a barrier whereas Ref. 6 considered it over a well. Furthermore, in Ref. 6 the transmission of a Gaussian wave packet, with a spread in energy, was investigated, which can be deconvoluted into a series of plane waves around an average wave number, while here the transmission of a plane wave with a well-defined energy or wave vector was studied. In principle, we could take the injected, reflected, and transmitted wave packages of Ref. 6, Fourier transform them, and obtain the transmission and reflection coefficients for each wave vector, i.e., for each energy, and add this difference to the manuscript. However, the aim here is not to present an exact comparison between these two approaches, which is unrealistic because of the different models used, but to show that we can simulate the Lorentz-force-induced asymmetric injection of electrons into the arms of the rings with a simple analytical calculation. We expect though that such a calculation will lead to some minor quantitative discrepancies in the transmission even if the approach of Ref. 6 is applied to a barrier, as in our case, due to the spread in the energy of the incident electrons.

\section{CONCLUDING REMARKS}

We evaluated the transmission through a mesoscopic ring, in the presence of an $\mathrm{AB}$ flux, using an $\mathbf{S}$ matrix that is not symmetric with respect to the two arms of the ring. All elements were expressed in terms of two parameters, $a$ and $\lambda$, the latter expressing the asymmetry through $\lambda=b / c$, with $b$, $c$ the pertinent elements of the $\mathbf{S}$ matrix. The determinant of the $\mathbf{S}$ matrix and the transmission are invariant under the change $\lambda \rightarrow 1 / \lambda$. Previous results of the literature pertaining to a symmetric $\mathbf{S}$ matrix were readily recovered for $\lambda=1$. The dependence on the parameter $\lambda$ disappears from the transmission [see Eqs. (6) and (7)], but not from the more general result given by Eq. (10) when the flux is zero. It is important mostly for a nonzero flux and, depending on the parameters, it modifies the results considerably as shown in Figs. 2 and 3, where results for $\lambda=1$ and $\lambda=3$ are contrasted.

We also evaluated the transmission when an asymmetry was introduced externally, i.e., when one scatterer was placed in one of the ring's arms. Of course this applies to both cases $\lambda=1$ and $\lambda \neq 1$. The transmission shows a rich structure as a function of the parameter $a, \phi$ (or wave vector), $\Phi / \Phi_{0}$, and the strength $T_{s}$ (cf Figs. 1-5). The results for different $a$ shown in Figs. 4 and 5 correspond to those for $\epsilon$ in Ref. 9 in which no results as a function of the flux or the strength $T_{s}$ were shown.

Importantly, results on rings of finite width could be mimicked in two ways. In one way we simply fitted the results of a heavy numerical treatment ${ }^{6}$ by using $\lambda=c /\left[1+\left(\Phi / \Phi_{0}\right)^{s}\right]$, with $s$ between 1 and 2. The two results are in good qualitative agreement, especially in the case when no scatterer was present in the arms, cf. Fig. 5. When a scatterer was present a real comparison could not be made due to the different nature of the scatterers involved, a barrier in our case, a Gaussian well in that of Ref. 6. Since the stronger the magnetic field, the stronger the asymmetry obtained in Ref. 6, it is natural to expect that it should be reflected in $\lambda$ even if our treatment applies only to rings whose width is much smaller than their radii and only the lowest (in the radial direction) energy level is occupied. As we saw the overall trend in $T$ vs $\Phi / \Phi_{0}$ was reproduced quite well though not all the details. We emphasize that this agreement cannot be obtained with an $S$ matrix that is symmetric with respect to both arms, i.e., for $\lambda=1$. 
Despite the agreement just mentioned above, the model has its limitations as was pointed out at the end of Sec. IV, regarding a further comparison between its results and those of Ref. 6, and at the end of Sec. III regarding a possible improvement of the agreement, upon using Eq. (10) with $\lambda$ $\neq 1$, between the reported experimental ${ }^{12}$ and theoretical results. Also, if the potentials in the two arms are different, complex interference patterns may result and the asymmetric injection, affecting only the amplitude of the transmission, is unlikely to completely describe the systems discussed in this paper.

In another way we used results of the literature that incorporate the width of the ring in the boundary conditions, though not rigorously, to determine the parameters $a$ and $\epsilon$ by comparing results for the transmission between different approaches. This made $a$ and $\epsilon$ depend on the energy and the width of the ring. In addition, through this comparison we demonstrated the equality $a^{2}=1-\epsilon$.

Another externally imposed asymmetry that could be considered is to have the lengths of the two arms unequal. This has been treated in Ref. 7 using a symmetric $\mathbf{S}$ matrix and, as expected, lead to a certain dephasing of the $\mathrm{AB}$ oscillations. A similar study, involving arms of unequal length and a 4 $\times 4 \mathbf{S}$ matrix in the presence of SOI but without scatterers in the arms, appeared recently. ${ }^{14}$

A possible extension of the theory presented here would be to consider in detail the real nature of the scatterers, barriers or wells, placed in one arm and have an explicit energy dependence in the transmission $\left(T_{s}\right)$ and reflection $\left(R_{s}\right)$ probabilities instead of taking them as parameters as we did. Another extension would be to consider a chain of rings with periodic modulations in the magnetic field or ring radius in analogy with a recent work on rings in the presence of SOI. ${ }^{15}$ A last extension concerns the determination $\lambda$. Since $\lambda$, like $\epsilon$ or $a$, is a parameter constrained only by the unitarity of the $\mathbf{S}$ matrix (it has to be real), it cannot be evaluated. The only way it could be determined is to compare two transmission results obtained by two different methods as we have done in Sec. V for the parameters $\epsilon$ and $a$. Presently, such results are not available. All these extensions are left for future work. We expect that our results, though incomplete in some respects, will be tested by appropriate experiments.

\section{ACKNOWLEDGMENTS}

This work was supported by the Flemish-Hungarian bilateral program, the Canadian NSERC Grant No. OGP0121756, the Flemish Science Foundation (FWO-VI), and the SANDiE EU network of excellence. O.K. is supported by the Marie Curie training project. O.K. and M.G.B. are also supported by the Hungarian Scientific Research Fund under Contract No. 48888.

\section{APPENDIX A}

In part (i) below we determine the elements of the $\mathbf{S}$ matrix and in part (ii) we solve the systems of equations $\boldsymbol{\alpha}_{2}^{\prime}$ $=\mathbf{S} \boldsymbol{\alpha}_{2}$ and $\boldsymbol{\alpha}_{1}^{\prime}=\mathbf{S} \boldsymbol{\alpha}_{1}$. (i) The unitarity of the $\mathbf{S}$ matrix [cf. Eq. (2)], leads to the following relations between its elements $a, b, c, d, e, f$ :

$$
\begin{gathered}
a^{2}+b^{2}+c^{2}=b^{2}+d^{2}+e^{2}=c^{2}+e^{2}+f^{2}=1, \\
a b+b d+c e=a c+b e+c f=b c+d e+e f=0 .
\end{gathered}
$$

To reduce the number of parameters and make the two arms not equivalent to each other, we take $b=\lambda c$. Then Eqs. (A2) give $e=-\lambda(a+d)$ and $f=-a-d \pm 1$. With $b=\lambda c$, Eqs. (A1) give $b= \pm \lambda\left(1-a^{2}\right)^{1 / 2} / \mu$, where $\mu=\left(\lambda^{2}+1\right)^{1 / 2}$. Obviously, 1 $-a^{2} \geqslant 0$, i.e., $|a| \leqslant 1$. Then Eqs. (A1) and $e=-\lambda(a+d)$ determine $d$ as $d=\left(\lambda^{2} a-1\right) / \mu^{2}$. Thus, all elements can be expressed in terms of $a$ and $\lambda$ and the result is given by Eq. (3). Reference 9 took $\lambda=1$ and instead of $a$, used the parameter $\epsilon$, $0 \leqslant \epsilon \leqslant 1 / 2$. The value $\epsilon=1 / 2$ corresponds to $a=0$ and $\epsilon=0$ to $a=1$.

(ii) For the right junction we obtain

$$
\begin{aligned}
& \alpha_{2}^{\prime}=b \beta_{2}+c \gamma_{2}, \\
& \beta_{2}^{\prime}=d \beta_{2}+e \gamma_{2}, \\
& \gamma_{2}^{\prime}=e \beta_{2}+f \gamma_{2} .
\end{aligned}
$$

Using Eqs. (A4) and (A5) we can write

$$
\left(\begin{array}{c}
\gamma_{2}^{\prime} \\
\gamma_{2}
\end{array}\right)=\mathbf{t}_{l 2}\left(\begin{array}{c}
\beta_{2} \\
\beta_{2}^{\prime}
\end{array}\right)
$$

where $\mathbf{t}_{l 2}$ is the matrix

$$
\mathbf{t}_{12}=\frac{1}{e}\left(\begin{array}{cc}
e^{2}-f d & f \\
-d & 1
\end{array}\right)
$$

Notice that $\operatorname{det}\left(\mathbf{t}_{l 2}\right)=1$.

For the left junction we obtain

$$
\begin{aligned}
& \alpha_{1}^{\prime}=a+b \beta_{1}+c \gamma_{1}, \\
& \beta_{1}^{\prime}=b+d \beta_{1}+e \gamma_{1}, \\
& \gamma_{1}^{\prime}=c+e \beta_{1}+f \gamma_{1} .
\end{aligned}
$$

Using Eqs. (A9) and (A10), we can write

$$
\left(\begin{array}{c}
\beta_{1}^{\prime} \\
\beta_{1}
\end{array}\right)=\frac{b}{e}\left(\begin{array}{c}
b e-d c \\
-c
\end{array}\right)+\mathbf{t}_{l 1}\left(\begin{array}{c}
\gamma_{1} \\
\gamma_{1}^{\prime}
\end{array}\right),
$$

where $\mathbf{t}_{l 1}$ is given by $\mathbf{t}_{l 2}$ with $d$ and $f$ interchanged.

The connection between the two junctions is made by writing

$$
\left(\begin{array}{c}
\beta_{2} \\
\beta_{2}^{\prime}
\end{array}\right)=e^{-i \theta_{1}} \mathbf{t}_{1}\left(\begin{array}{l}
\beta_{1}^{\prime} \\
\beta_{1}
\end{array}\right),
$$

for the amplitudes in the upper arm, and

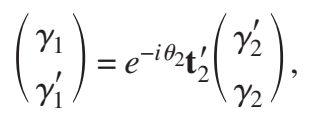

for the amplitudes in the lower arm, where $\mathbf{t}_{1}$ and $\mathbf{t}_{2}$ are the matrices associated with the first and second scatterer given 
by Eq. (A7). Combining Eq. (A6) and Eqs. (A11)-(A13) we can write

$$
\mathbf{P}\left(\begin{array}{l}
\beta_{1}^{\prime} \\
\beta_{1}
\end{array}\right)=-\frac{b}{e}\left(\begin{array}{c}
b e-c d \\
-c
\end{array}\right)
$$

with

$$
\mathbf{P}=\mathbf{t}_{l 1} e^{-i \theta_{2}} \mathbf{t}_{2}^{\prime} \mathbf{t}_{l 2} e^{-i \theta_{1}} \mathbf{t}_{1}-\mathbf{1},
$$

where $\mathbf{1}$ is the unit matrix. The transmitted amplitude $\alpha_{2}^{\prime}$ is obtained from Eqs. (A3) and (A4) $\left(\Lambda=\lambda^{2}+1\right)$,

$$
\alpha_{2}^{\prime}=\left[\left(1-a^{2}\right) \Lambda\right]^{1 / 2}\left(\beta_{2}-\beta_{2}^{\prime}\right) / \lambda(a+1),
$$

and the coefficients $\beta_{2}$ and $\beta_{2}^{\prime}$ from Eq. (A9) after solving Eq. (A11) for $\beta_{1}$ and $\beta_{1}^{\prime}$. Inserting these values of $\beta_{2}$ and $\beta_{2}^{\prime}$ in Eq. (A16) gives Eq. (6) of Sec. III.

\section{APPENDIX B}

Griffith's boundary conditions applied at a junction between the leads and the ring, e.g., at the black triangles in
Fig. 1, are (i) the continuity of the wave function and (ii) the continuity of the flux. For a ring of finite width one modifies condition (ii) by adding a term $2 \nu \psi / W$ to the left $\operatorname{side}^{13}$ so that it reads

$$
\sum_{i=1}^{N} \frac{\partial \psi}{\partial x_{i}}+\frac{2 \nu \psi}{W}=0,
$$

where $N$ is the number of the legs at a junction $(N=3$ here) and $\nu$ a parameter of order 1 to be determined from a comparison with an exact numerical result.

We have applied these conditions to a ring of finite width connected to two leads of the same width. On each line segment the wave function is given by $\psi=A_{i} \exp \left(i k x_{i}\right)$ $+B_{i} \exp \left(-i k x_{i}\right)$. The inner and outer radii of such a ring are $R-W / 2$ and $R+W / 2$, respectively. Assuming nothing is incident from the right of the right junction, and setting $\mu$ $=4 \nu^{2} / k^{2} W^{2}$ we obtain the transmission, not given in Ref. 13, as

$$
T=16 /\left[(\mu+1)(\mu+9) \sin ^{2}(\pi k R)+16\right] .
$$

${ }^{1}$ Y. Aharonov and D. Bohm, Phys. Rev. 115, 485 (1959).

${ }^{2}$ Y. Aharonov and A. Casher, Phys. Rev. Lett. 53, 319 (1984).

${ }^{3}$ M. V. Berry, Proc. R. Soc. London, Ser. A 392, 45 (1984).

${ }^{4}$ Y.-S. Yi, T.-Z. Qian, and Z.-B. Su, Phys. Rev. B 55, 10631 (1997); D. Frustaglia and K. Richter, ibid. 69, 235310 (2004); S. Souma and B. K. Nikolic, ibid. 70, 195346 (2004).

${ }^{5}$ T. Choi, S. Y. Cho, C. M. Ryu, and C. K. Kim, Phys. Rev. B 56, 4825 (1997); B. Molnár, F. M. Peeters, and P. Vasilopoulos, ibid. 69, 155335 (2004); X. F. Wang and P. Vasilopoulos, ibid. 72, 165336 (2005).

${ }^{6}$ B. Szafran and F. M. Peeters, Phys. Rev. B 72, 165301 (2005); Europhys. Lett. 70, 810 (2005).

${ }^{7}$ C. Benjamin and A. M. Jayannavar, Phys. Rev. B 65, 153309 (2002); S. Bandopadhyay, P. S. Deo, and A. M. Jayannavar, ibid. 70, 075315 (2004); M. Büttiker, SQUID '85, Superconducting Quantum Interference Devices and their Applications, edited by H. D. Hahlbohm and H. Lübbig (Walter de Gruyter, Berlin, 1985), p. 529.

${ }^{8}$ A. Yacoby, M. Heiblum, D. Mahalu, and H. Shtrikman, Phys. Rev. Lett. 74, 4047 (1995); G. Cernicchiaro, T. Martin, K. Hasselbach, D. Mailly, and A. Benoit, ibid. 79, 273 (1997); R. Schuster, E. Buks, M. Heiblum, D. Mahalu, V. Umansky, and H. Shtrikman, Nature (London) 385, 417 (1997); S. Pedersen, A. E. Hansen, A. Kristensen, C. B. Sorensen, and P. E. Lindelof,
Phys. Rev. B 61, 5457 (2000); M. Koenig, A. Tschetschetkin, E. M. Hankiewicz, J. Sinova, V. Hock, V. Daumer, M. Schaefer, C. R. Becker, H. Buhmann, and L. W. Molenkamp, Phys. Rev. Lett. 96, 076804 (2006); Y. Ji, Y. Chung, D. Sprinzak, M. Heiblum, D. Mahalu, and H. Shtrikman, Nature (London) 422, 415 (2003).

${ }^{9}$ M. Büttiker, Y. Imry, and M. Ya. Azbel, Phys. Rev. A 30, 1982 (1984).

${ }^{10}$ Y. Gefen, Y. Imry, and M. Ya. Azbel, Phys. Rev. Lett. 52, 129 (1984).

${ }^{11}$ We follow the usual approach of Refs. 6, 7, 9, and 10, in which $\phi(E)$ is an independent parameter with $E$ the Fermi level. In a more refined approach the Fermi level of the ring depends on whether the number of the particles is even or odd [see C. H. Wu and G. Mahler, Phys. Rev. B 43, 5012 (1991)].

${ }^{12}$ S. Pedersen, A. E. Hansen, A. Kristensen, C. B. Sorensen, and P. E. Lindelof, Phys. Rev. B 61, 5457 (2000).

${ }^{13}$ K.-K. Voo, S.-C. Chen, C.-S. Tang, and C.-S. Chu, Phys. Rev. B 73, 035307 (2006).

${ }^{14}$ U. Aeberhard, K. Wakabayashi, and M. Sigrist, Phys. Rev. B 72, 075328 (2005).

${ }^{15}$ B. Molnár, P. Vasilopoulos, and F. M. Peeters, Phys. Rev. B 72, 075330 (2005). 